\title{
Recent advances in the treatment of erectile dysfunction
}

\author{
David F Mobley, ${ }^{1}$ Mohit Khera, ${ }^{2}$ Neil Baum ${ }^{3}$
}

'Department of Urology, WeillCornell Medicine, Houston,

Texas, USA

2Department of Urology, Baylor College of Medicine, Houston, Texas, USA

${ }^{3}$ Department of Urology, Tulane Medical School, New Orleans, Louisiana, USA

\section{Correspondence to} Dr David F Mobley, Department of Urology, Weill-Cornell Medicine, 18300 Katy Fwy, Ste 325, Houston 77094, TX, USA; mobleyresearch@gmail.com

Received 14 March 2016 Revised 21 February 2017 Accepted 23 April 2017 Published Online First 27 July 2017

\section{ABSTRACT}

Erectile dysfunction (ED) is one of the most common conditions affecting middle-aged and older men. Nearly every primary care physician, internist and geriatrician will be called upon to manage this condition or to make referrals to urologists, endocrinologists and cardiologists who will assist in the treatment of ED. This article will briefly discuss the diagnosis and management of ED. In addition, emerging concepts in ED management will be discussed, such as the use of testosterone to treat ED, the role of the endothelium in men with ED and treating the partner of the man with ED. Finally, future potential therapies for ED will be discussed.

\section{INTRODUCTION}

Nearly every primary care physician, internist and geriatrician now understand that many older men retain an interest in sexual activity as they age. Some primary care physicians think that sexual potency in older men is the norm, and that if it is lacking, it is 'all in the head.' This viewpoint has not been supported by current literature. The Massachusetts Male Aging Study (MMAS) found that 52\% of men between 40 and 70 years old reported having some form of erectile dysfunction (ED). ${ }^{1}$ The reality is that ED is a natural part of ageing and that the prevalence increases with age. In the MMAS, they found that roughly $50 \%$ of men at 50 years old, $60 \%$ of men at 60 years old and $70 \%$ of men at 70 years old had ED. Thus, nearly all men who live long enough should develop ED. The myths that surround the problems of impotence or ED confound the attempts of patients to receive treatment and the attempts of physicians to help them. ${ }^{1}$

Many factors can contribute to sexual dysfunction in older men, including physical and psychological conditions, comorbidities and the medications used to treat them. Aspects of an ageing man's lifestyle and behaviour and androgen deficiency, most often decreasing testosterone levels, may affect sexual function as well. A study of men between the ages of 30 and 79 years showed that $24 \%$ had testosterone levels below $300 \mathrm{ng} / \mathrm{dL}$ and 5.6\% had symptomatic androgen deficiency. ${ }^{2}$

The percentage of men who engage in some form of sexual activity decreases from $73 \%$ for men aged $57-64$ years to $26 \%$ for men aged $75-85$ years. ${ }^{3}$ For some men, this constitutes a problem, but for others it does not. The aetiology for this decline in sexual activity is multifactorial and is in part due to the fact that most of the female partners undergo menopause at 52 years of age with a significant decline in their libido and desire to engage in sexual activity. A study by Lindau and colleagues ${ }^{3}$ that examined sexuality in older Americans showed that $50 \%$ of the men in a probability sample of more than 3000 US adults reported at least one bothersome sexual problem and 33\% had at least two such problems. ${ }^{3}$ This article will review the normal changes that occur with ageing, factors that influence these changes, individual variations and perspectives, and the available treatment options for ED and androgen deficiency.

\section{BRIEF HISTORY OF THE TREATMENT OF ED}

Impotence treatments were discussed in the oldest Chinese text, The Yellow Emperor's Classic of Internal Medicine, which describes traditional Chinese medicine during the time of the Yellow Emperor's rule which ended around 2600 BC. One of the treatments for impotence discussed is a potion with 22 ingredients. ${ }^{4}$

Nearly 1000 years later, the Egyptian Papyrus Ebers, a medical Egyptian document dated 1600 $\mathrm{BC}$, describes a cure for impotence in which baby crocodile hearts were mixed with wood oil and applied topically to the penis. ${ }^{4}$

In 1973, Dr Brantley Scott from Baylor College of Medicine reported on the implantable inflatable prosthesis that urologists still use today. ${ }^{5}$

The major breakthrough occurred in 1998 when sildenafil became the first oral drug to be approved to treat ED. ${ }^{4}$ This was followed by the use of tadalafil and vardenafil as similar phosphodiesterase-5 inhibitor oral medications for treating ED in $2003 .{ }^{4}$

\section{OVERVIEW OF PHYSIOLOGICAL CHANGES AND OTHER RISK FACTORS}

As with most other organ system in the human body, changes and loss of function is normal consequence of the ageing process. This is also true of the endocrine system, specifically the levels of testosterone production from the Leydig cells of the testicle. Accompanying the decrease in testosterone is a decrease in erections which also has a component in decrease in the blood supply to the penis making erection not as frequent and not as rigid compared with a young man's erectile function. Although these changes are in itself not life threatening, they can impact a man's relationship with his partner, and also ED may be a harbinger of other undiagnosed conditions such as coronary artery disease (CAD), hypercholesterolaemia or diabetes mellitus. ${ }^{6}$

ED is defined as the inability to achieve a full erection or the inability to maintain an erection adequate for sexual intimacy. Other types of sexual 
dysfunction such as premature ejaculation and low libido may occur; however, the most common and disruptive problem in men is ED. Although most men will experience periodic episodes of ED, these episodes tend to become more frequent with advancing age.

Medical conditions, such as hypertension, diabetes mellitus, and cardiovascular disease (CVD), and psychological conditions, such as depression and anxiety, also contribute to sexual dysfunction in middle-aged or elderly men. CVD and hypertension cause a narrowing and hardening of the arteries, leading to reduced blood flow to the corporal bodies, which is essential for achieving an erection. Diabetes is a common aetiology of sexual dysfunction, because it can affect both the blood vessels and the nerves that supply the penis. Men with diabetes are four times more likely to experience ED, and on average, experience ED 15 years earlier than men without diabetes. ${ }^{7}$ Obesity is also correlated to the development of several types of dysfunction, including a decrease in sex drive and an increase in episodes of ED. ${ }^{8}$

There are hundreds of medications that have the side effect of ED and/or decreased libido. Examples of drugs implicated as a cause of ED include hydrochlorothiazides and beta-blocking agents. Medications used to treat depression, particularly the SSRIs such as citalopram (Celexa), escitalopram (Lexapro), fluoxetine (Prozac, Prozac Weekly, Sarafem), fluvoxamine (Luvox, Luvox CR), paroxetine (Paxil, Paxil CR, Pexeva) and sertraline (Zoloft), may also contribute to ED. ${ }^{9}$ Bupropion (Wellbutrin) which has a predominant effect on blocking the reuptake of dopamine is an antidepressant with lower incidence of ED. ${ }^{10}$ The side effects of 5ARIs occurring in fewer than 5\% of patients can include gynaecomastia, ED, loss of libido and ejaculatory dysfunction. ${ }^{11}$

Men being treated for prostate cancer with treatments such as radical prostatectomy, radiation therapy or the use of Lutenizing hormone-releasing hormone (LHRH) agonists and antagonists can expect that ED may accompany these treatments. ${ }^{12}$

Smoking, excessive use of alcohol and illicit drugs are also associated with ED. ${ }^{13}$ A study in 2005 suggests that ED is not only more likely among men who smoked compared with those who never did, but that in younger men with ED, cigarette smoking is very likely the cause of their impotence. ${ }^{14} 15$

Also, it is important to remember that there are psychogenic issues such as performance-related issues, traumatic past experiences, relationship problems, anxiety, depression and stress that can certainly cause or be considered a comorbid condition contributing to ED.

Bullet point: Ageing and comorbidities as well as polypharmacy factor greatly influence the development of ED.

\section{ROLE OF THE ENDOTHELIUM IN ED}

The role of the endothelium in ED has been noted for a number of years and the overlapping of ED and other conditions, especially coronary heart disease, CVD, affecting endothelial function/dysfunction, is clearly present. The endothelial cell is now known to affect vascular tone and impact the process of atherosclerosis, and impacting ED, CVD and peripheral vascular disease. ${ }^{16}$

The role of the endothelium in erectile function became clearer with the observation that the phosphodiesterase type 5 (PDE5) inhibitor, sildenafil, enhanced erectile function. Erection occurs with the release of nitric oxide (NO) from the vascular endothelial cells. ${ }^{17}$ The reduction in endothelial cell production of NO results in the negative impact on the smooth muscles in the corporal bodies and results in less relaxation of the smooth muscle cells with decrease in blood supply and resulting ED. A similar phenomenon is well known to impact the coronary arterial system resulting in CVD.

It is important to understand that ED is frequently, if not usually, directly related to endothelial dysfunction, and that the release of NO by the vasculature of the penile arteries is directly related to the function of intact, healthy endothelium. In the face of endothelial dysfunction, the process of erection fails to occur in a normal fashion. ${ }^{16}$

\section{THE ASSOCIATION OF ED, CVD AND ENDOTHELIAL DYSFUNCTION}

The pathogenesis of organic ED is related to dysfunction of the endothelium. Endothelial cells can become injured through a variety of mechanisms, most of which cause oxidative stress on the tissues. Many of these causes of oxidative stress are related to lifestyle issues which lead to hypertension, diabetes and dyslipidaemia (figure 1). Endothelial cell dysfunction results in reduction of endothelium-dependent vasorelaxation as well as increased adhesion of leukocytes to the endothelium. Endothelial cell injury then leads to a variety of sequelae, including ED, other types of vasoconstriction, atherosclerosis and thrombus formation. $^{18}$

The association of CVD and ED was noted in 1997 as one analysed the results of the MMAS. In this landmark study, 1709 men aged 40-70 years were enrolled between 1987 and 1989 . A follow-up some 10 years later revealed a striking relationship between ED and CVD. In this study, it became clear that the risk factors for ED were very similar to those of CVD, such as diabetes mellitus, smoking and dyslipidaemia. ${ }^{18}$

Since endothelial dysfunction, CVD and ED are closely associated in epidemiological studies, the question for clinicians is whether to recommend the man presenting with ED undergo a cardiovascular (CV) evaluation. Clearly, based on numerous studies, ED can be considered at least a 'marker' for possible further vascular disease or CVD. ${ }^{15}$ In their report, Vlachopoulos and coworkers make the point that the man presenting with ED, the clinician, is offered an opportunity to attempt to improve the health of the man by addressing lifestyle modification, and consider further vascular evaluation owing to the clear relationship between endothelial dysfunction, ED and CVD. ${ }^{19}$

Watts and coworkers, in their review article, make several points about this ED/CAD nexus. Endothelial dysfunction is present in both CVD and ED, and is linked through the NO mechanism. The authors note that PDE5 inhibitors improve endothelial function and have a salutary effect on both CVD and ED. Both ED and cardiac disease respond to modifications in lifestyle as well as pharmacologic manipulation. These authors also report that the presence of ED gives the clinician an opportunity to assess CVD and prevention as well. ${ }^{20}$

Due to the fact that ED and CVD share many of the same risk factors, it is not surprising that there is a high incidence of ED in men who present with CVD. A study by Montorsi et al found that ED was present in roughly $50 \%$ of patients with acute chest pain and confirmed CAD on angiography. ${ }^{21}$

In this study, ED proceeded CVD in almost $70 \%$ of cases. Similarly, many men with ED have been found to have pre-existing CVD. A study by Vlachopoulos et al evaluated the incidence of asymptomatic CVD in 50 men with ED. ${ }^{22}$ These authors found that $19 \%$ of men with ED had asymptomatic CVD. Similarly, Mulhall and colleagues found that $20 \%$ of men presenting with 


\section{Common Link Between ED and Cardiovascular Disease: Endothelial Injury}

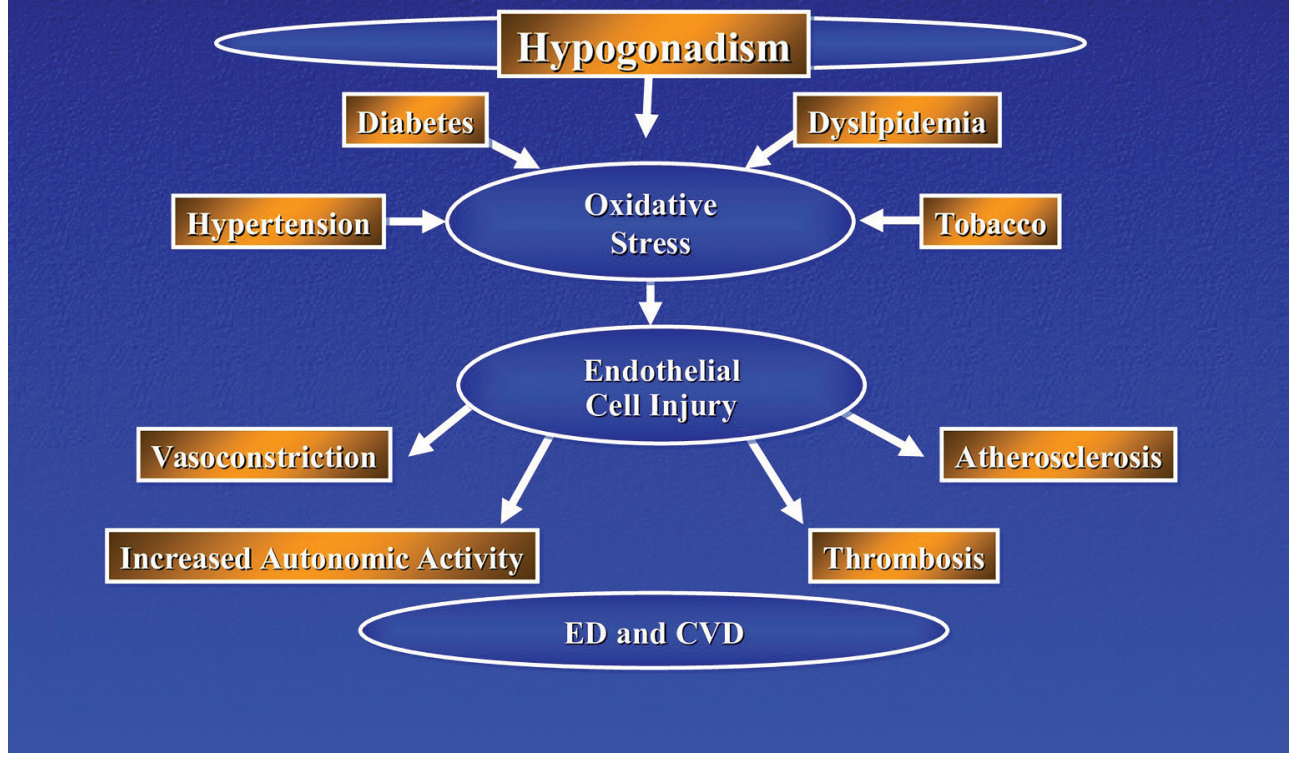

Figure 1 Link between oxidative stress, endothelial dysfunction and ED. CVD, cardiovascular disease; ED, erectile dysfunction.

ED and vascular insufficiency on penile duplex had asymptomatic CVD. ${ }^{23}$

These findings suggest that patients who present with ED and CV risk factors should be evaluated for silent CVD and should undergo a thorough CV evaluation.

Men with new onset ED and no signs or symptoms of CVD are at increased risk for experiencing a CV event in the subse-

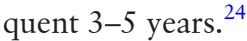

Men in their 40s with ED have a 50-fold increased risk of CVD and men in their 20s and 30s have a sevenfold increased CV risk. ${ }^{25}$

A meta-analysis of 36744 men with ED in 12 prospective cohort studies found that the presence of ED significantly increased the risk of CVD, CAD, stroke and all-cause mortality, and the presence of ED was an independent risk factor for CVD. Ponholzer et al found that men with moderate to severe ED had a $65 \%$ increased relative risk for developing symptomatic CAD compared with men who did not have ED. ${ }^{26}$

The Prostate Cancer Prevention Trial was a landmark study by Thompson et al that prospectively assessed the time to developing CVD after the diagnosis of ED. There were 4247 men with no ED at study entry; 2420 developed incident ED (defined as the first report of ED of any grade) over 5 years. Those men that developed ED had a 1.45 -fold higher probability of experiencing a $\mathrm{CV}$ event compared with men who did not develop ED. ${ }^{27}$

The severity of ED has been correlated with the extent of CVD. Banks et al reported that the risk of future CV events increased progressively according to ED severity. ${ }^{28}$ This was shown in both men with and without known CVD at baseline and after controlling for confounders. Solomon and colleagues found an inverse correlation between international index of erectile function (IIEF) scores and plaque burden seen on coronary angiography. ${ }^{29}$ In addition, Yaman et al demonstrated a significant correlation between ED severity on IIEF questionnaires and coronary artery calcification. ${ }^{30}$

Finally, Dr Montorsi et al found that severe ED was more common in patients with multivessel coronary involvement as compared with those with single-vessel disease (31\% vs $12.5 \%$; $\mathrm{p}<0.01)$. They also found a significant inverse relationship between the extent of CVD and IIEF scores. ${ }^{31}$

Normal erectile function depends on the release of $\mathrm{NO}$ and endothelial-dependent vasodilation of the penile arteries. The 'artery size' hypothesis, first described by Dr Montorsi, offers an explanation why men are more likely to develop ED before a myocardial infacrtion. It is believed that atherosclerosis affects all vascular beds equally but smaller arteries are more likely to become occluded than larger arteries. ${ }^{31} 32$ The penile arteries are $1-2 \mathrm{~mm}$ while the coronary arteries are $3-4 \mathrm{~mm}$. Thus, the same degree of endothelial dysfunction and atherosclerosis is more likely to occlude blood flow in the penile arteries compared with the coronary arteries. The penile arteries therefore serve as a sensitive indicator for subsequent CVD. This theory is supported by the fact that ED occurs approximately 3 years prior to cardiac symptoms in virtually all patients with chronic coronary syndrome whereas patients with acute coronary syndrome have a much lower prevalence of sexual dysfunction. ${ }^{32}$

Bullet point: The relationship between the risk factors for CVD and ED are intimately interwoven, one with the other. Clinicians need to factor this in when counselling the man with ED.

\section{TESTOSTERONE AND ERECTILE FUNCTION}

Among the phenomena in the ageing man are a decrease in erectile function and testosterone levels. Add to these, increased risk for CVD, muscle wasting, decrease in bone density and libido, with all of these factors having an interplay with testosterone metabolism. ${ }^{33}$ Androgens play a key role in maintaining erectile 
function through four main mechanisms. Androgen deprivation has been shown to result in impairment of NO synthase release, altered PDE5 expression and activity, impaired cavernosal nerve function, and contribution to veno-occlusive disease in the penis. ${ }^{34}$ The role of testosterone replacement therapy (TRT) as a potential to improve erectile function in the man with ED remains an issue for patient and physicians who are comfortable treating androgen deficiency which include primary care physicians and specialists. Androgens are known to have a significant impact on the function of the smooth musculature within the corpus spongiosum. ${ }^{35}$

The observation that TRT enhances the efficacy of PDE5 inhibitors in hypogonadal men taking these therapies with suboptimal response to the PDE5 inhibitors alone has been reported. ${ }^{33}$ In addition, investigators have demonstrated that TRT in hypogonadal men can improve erectile function even without the benefit of PDE5 inhibitors. ${ }^{33}$ In addition, guidelines for managing ED in hypogonadal men by the European Association of Urology recommend controlling the man to a eugonadal state prior to initiation of PDE5 inhibitor therapy. ${ }^{36}$ Testosterone measurement consists of a serum specimen which should be ideally obtained in the morning because of the normal diurnal variation of testosterone which is at its peak in the morning. Since TRT is relatively safe, and men can potentially see an improvement in erectile function, it seems prudent to consider this issue when presented with a patient suffering from ED.

\section{SAFETY OF TRT}

Recently, the US Food and Drug Administration (FDA) has issued a safety announcement regarding TRT. In part it reads 'The benefit and safety of these medications have not been established. We are also requiring these manufacturers to add information to the labeling about a possible increased risk of heart attacks and strokes in patients taking testosterone., ${ }^{, 37}$

In their extensive review, Bassil and coworkers summarise the benefits and risks, with benefits such as improvement of sexual function, bone density, muscle strength, cognition and overall improvement in quality of life. Among the risks that have been suggested include erythrocytosis, liver toxicity, worsening of sleep apnoea and cardiac function, possibly increasing symptoms of benign prostatic hyperplasia (BPH). They also note that although a possibility of stimulation of prostate cancer has been hypothesised, no scientific or clinical evidence exists to this possible risk. ${ }^{38}$

Erythrocytosis has been noted in men on TRT, and should be monitored every 6-12 months depending upon the patients' response to changes in haematocrit levels. For mild elevations, the dosage of testosterone can be decreased or the interval of using the medication can be increased. With the haematocrit greater than $50 \%$, decisions to temporarily discontinue the medication or periodic phlebotomy may be indicated. ${ }^{38}$

Liver toxicity has generally been associated with oral forms of TRT, but does not appear to be a risk in injection or transdermal therapy. ${ }^{39}$ In patients with marked obstructive sleep apnoea or untreated severe congestive heart failure, these issues should be resolved before initiating TRT. ${ }^{37}$

Clinicians should be aware of these possible detrimental effects of TRT and the possible contraindications, and to prescribe and monitor in an appropriate manner, which may vary from patient to patient.

Bullet point: In men with ED, assessing androgen status is a simple evaluation, and therapy when indicated can be beneficial, and in most men, safe.

\section{CURRENT TREATMENT OPTIONS}

As is true in so many medical conditions, lifestyle modifications, considered first-line therapy, can have a salutary effect in ED management, and men should be encouraged to make the necessary changes to the benefit of their sexual function and to their overall health as well. Despite the benefits of behaviour modification, men presenting with ED want the physician to help with measures that can have an immediate impact.

The options for management beyond behaviour modification include TRT, PDE5 inhibitors, intracavernosal injection therapy, vacuum constriction devices (VCDs), intraurethral prostaglandin suppositories and surgical placement of a penile prosthesis. $^{40}$

PDE5 inhibitors, the primary second-line therapy, have been the mainstay of ED treatment since the release of sildenafil (Viagra) in 1998, with the subsequent development of many others, and still more in the development stage. These medications do improve erectile quality for the majority of men, and they work by enhancing blood flow in the corpora cavernosa. These medications are generally used on demand and need to be taken about an hour before sexual intimacy. Tadalafil (Cialis) is longer acting and does come in a daily preparation potentially eliminating the 'on-demand' need. The daily dosing of tadalafil, 2.5-5 mg $\backslash$ day, has also been approved by the FDA for treatment of symptoms of $\mathrm{BPH} .{ }^{41}$ PDE5 inhibitors are contraindicated in men taking nitrates, but otherwise PDE5 inhibitors are very safe and effective. When PDE5 inhibitors are coadministered with nitrates, pronounced systemic vasodilation and severe hypotension are possible. Many patients with ED are elderly and have the same risk factors as patients with CAD, so these drug combinations are commonly considered or encountered in clinical practice. $^{42}$

It is important for clinicians prescribing these drugs to make the patient aware of the action of the drugs especially the fact that they do not result in an immediate erection, and that they do not cause an erection without sexual stimulation. There is frequently a great expectation when men begin using these drugs and it is wise to temper their enthusiasm and explain they do not work immediately, and may not work every time, but also let the patient know that if these drugs do not work, there are other options.

Besides PDE5 inhibitors and among second-line therapies are VCDs which are clear plastic chambers placed over the penis, tightened against the lower abdomen with a mechanism to create a vacuum inside the chamber. This directs blood into the penis. If an adequate erection occurs inside the chamber, the patient slips a small constriction band off the end of the VCD and onto the base of the penis. An erection beyond $30 \mathrm{~min}$ is not recommended. These devices can be a bit cumbersome, but are very safe. ${ }^{40}$

Another second-line therapy is the use of alprostadil, either intracavernosal or intraurethral. Two products are available for direct injection, Caverject (Pfizer) or Edex (Actient). A small needle is used to inject the medication into the lateral aspect of the penis through a small-gauge needle.

Response is dose related and usually occurs within 10-15 min, and does not require stimulation. The intraurethral preparation, medicated urethral suppository for erection (MUSE), consists of a tiny pellet of drug inserted into the urethral meatus. Response is also dose related, and onset similar to the cavernosal preparations. 


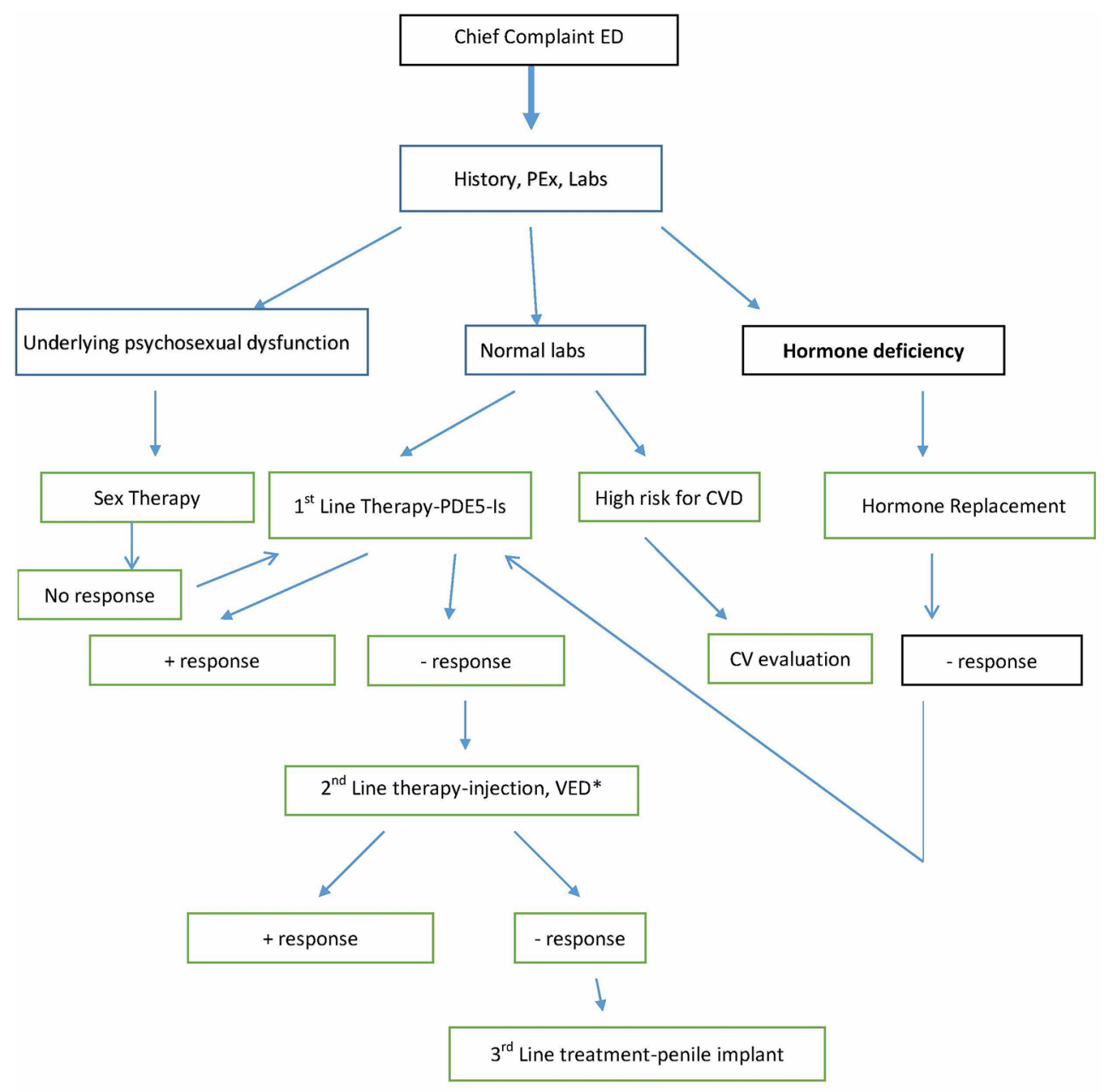

*vacuum erection device

Figure 2 Algorithm for evaluating and managing the patient with ED. CV, cardiovascular; CVD, cardiovascular disease; ED, erectile dysfunction.

\section{PSYCHOSEXUAL COUNSELLING}

Psychosexual counselling, or sex therapy, is an appropriate recommendation especially for men who are experiencing discord with their partner especially if the conflict is related to the man's ED. Counselling usually consists of 5-20 sessions with counsellor. It is our recommendation that referral doctors treating men with ED make a referral to a psychotherapist or sex therapist who is certified by AASECT (American Association of Sexuality Educators, Counselors and Therapists) of certified sexuality educator. ${ }^{43}$

Studies have shown that for men with stress-related ED, when the partner is involved in the therapy, the problem is resolved $50 \%-70 \%$ of the time. When the man must go through counselling alone, the results are less successful. Psychosexual counselling is unlikely to be effective if a man drops out of treatment after just one or two sessions. ${ }^{44}$

In patients who either fail to respond to first or second-line therapy, or are not interested in the conservative therapies, penile prosthesis implantation is available. Malleable and rigid implants were available for many years, but in 1973 the world of penile prosthetics took a giant leap forward with the advent of the inflatable penile implant. Most implants done nowadays are of the inflatable variety. Adverse events including malfunction and infection are rare, and patient satisfaction is very high. ${ }^{45}$
For an algorithm to facilitate evaluation and treatment of patients with ED, see figure 2 .

\section{FUTURE THERAPIES}

On the horizon is gene therapy that would deliver genes that produce products or proteins that may not be functioning properly in the penile tissue of men with ED. Replacement of these proteins may result in improvement in erectile function. Experimental animal models have demonstrated improvement in erectile function with gene therapy. Human studies may also demonstrate success with this therapy. Gene therapy may take a long time for regulatory approval and public acceptance.

The first stem cell study for the treatment of ED was published in 2004. This study used embryonic stem cells to treat ED. At this time, there is a total of 36 published basic studies assessing stem cell therapy for ED, with two clinical trials. The mechanism of action of stem cells is to generate angiogenesis with subsequent increase in cavernosal smooth muscle cells within the corporal bodies. ${ }^{46}$

Another potential new treatment consists of penile low-intensity shock wave lithotripsy. This consists of 1500 shocks twice a week for 3-6 weeks. The purpose is to stimulate neovascularisation to the corporal bodies with improvement in penile blood 
flow and endothelial function. The use of low-intensity shock wave lithotripsy may convert PDE5 inhibitor non-responders to responders. $^{47}$

Finally, there are NO-releasing polymers that are capable of delivering $\mathrm{NO}$ in a pharmacologically useful way. Such compounds include compounds that release NO upon being metabolised and compounds that release NO spontaneously in aqueous solution. Initial animal studies suggest that cavernosal injections of NO polymers can significantly improve erectile function. $^{48}$

\section{Current research questions}

Many areas in the field of ED remain ripe for further investigation:

- Can we develop a simple yet accurate method to distinguish organic from psychogenic ED?

- What are truly the norms for testosterone levels in men and could we better determine which might actually benefit, and thus, should receive TRT?

- What is the future of stem-cell therapy in the treatment of men with $\mathrm{ED}$

\section{Main messages}

- The risk of developing ED increases with age.

- Nearly every primary care physician, internist and geriatrician will be treating men with ED.

- The recent shift in the management and evaluation of ED, with primary care physicians replacing urologists in the forefront of ED diagnosis and therapy, has been a welcome and timely change.

- It is likely to improve ED management and benefit a large number of men, particularly in terms of recognising ED as a sentinel of vascular disease.

\section{Self assessment questions}

Please answer true and false to the below statements.

1. Men in their 40s with erectile dysfunction (ED) compared with men without a history of ED have an increased risk of developing cardiovascular disease (CVD) in 5 years.

2. Men presenting with ED should have consideration for a cardiovascular work-up as significant numbers of these men have occult or asymptomatic heart or vascular disease.

3. Endothelial dysfunction is more common in men with ED than in men with CVD.

4. The most likely explanation for men developing ED prior to developing CVD is that the penile arteries are much smaller than the coronary arteries and the smaller penile arteries are likely to be occluded before the coronary arteries are significantly narrowed and become symptomatic.

5. All men receiving testosterone replacement need to have periodic measurement of haemoglobin and haematocrit to monitor for erythrocytosis.

Contributors DFM and NB wrote the majority of the manuscript. MK wrote the section on testosterone and made review comments and approved the final manuscript along with the attached figure.

Competing interests None declared.

Provenance and peer review Not commissioned; externally peer reviewed. (c) Article author(s) (or their employer(s) unless otherwise stated in the text of the article) 2017. All rights reserved. No commercial use is permitted unless otherwise expressly granted.

\section{REFERENCES}

1 Feldman HA, Goldstein I, Hatzichristou DG, et al. Impotence and its medical and psychosocial correlates: results of the Massachusetts Male Aging Study. J Urol 1994:151:54-61.

2 Araujo $A B$, Esche GR, Kupelian V, et al. Prevalence of symptomatic androgen deficiency in men. J Clin Endocrinol Metab 2007:92:4241-7.

3 Lindau ST, Schumm LP, Laumann EO, et al. A study of sexuality and health among older adults in the United States. N Eng/ J Med 2007:357:762-74.

4 Shah J. Erectile dysfunction through the ages. BJU Int 2002;90:433-41.

5 Mobley D. Early history of inflatable penile prosthesis surgery. Asian J Androl 2015; 17:225-9.

6 Roumeguère T, Wespes E, Carpentier Y, et al. Erectile Dysfunction is associated with a high prevalence of hyperlipidemia and coronary Heart Disease Risk European Urology.44:355-9.

7 Klein R, Klein BE, Lee KE, et al. Prevalence of self-reported erectile dysfunction in people with long-term IDDM. Diabetes Care 1996;19:135-41.

8 Larsen SH, Wagner G, Heitmann BL. Sexual function and obesity. Int J Obes 2007:31:1189-98.

9 McWaine DE, Procci WR. Drug-induced sexual dysfunction. Med Toxicol Adverse Drug Exp 1988;3:289-306.

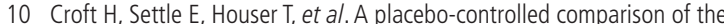
antidepressant efficacy and effects on sexual functioning of sustained-release bupropion and sertraline. Clin Ther 1999:21(4):643-58.

11 Janeway M, Baum N. Managing the enlarged prostate gland in elderly men. Clinical Geriatrics http://www.consultant360.com/articles/managing-enlarged-prostate-glandelderly-men.

12 Kumar RJ, Barqawi A, Crawford ED. Adverse events associated with hormonal therapy for prostate Cancer. Rev Urol 2005;7 Suppl 5:S37-S43.

13 Aksam A, Yassin A, Saad F. Testosterone and erectile dysfunction. J Andrology 2008;29.

14 Gades NM, Nehra A, Jacobson DJ, et al. Association between smoking and erectile dysfunction: a population-based study. Am J Epidemiol 2005;161:346-51.

15 Mobley D, Baum N. Smoking: it's impact on urologic conditions. Rev Urology 172015

16 Stein RA. Endothelial dysfunction, erectile dysfunction, and coronary heart disease: the pathophysiologic and clinical linkage. Rev Urol 2003;5(Suppl 7):S21-S27.

17 Andersson K, Stief C. Penile erection and cardiac risk: pathophysiologic and pharmacologic mechanisms. Am J Cardiol 2000;86:23-6.

18 Feldman HA, Johannes CB, Derby CA, et al. Erectile dysfunction and coronary risk factors: prospective results from the Massachusetts male aging study. Prev Med 2000;30:328-38

19 Vlachopoulos C, loakeimidis N, Terentes-Printzios D, et al. The triad: erectile dysfunction-endothelial dysfunction-cardiovascular disease Curr Pharm Des. 2008:14:3700-14.

20 Watts GF, Chew KK, Stuckey BG et al. The erectile-endothelial dysfunction nexus: new opportunities for cardiovascular risk prevention. Nat Clin Pract Cardiovasc Med 2007;4:263-73.

21 Montorsi F, Briganti A, Salonia A, et al. Erectile dysfunction prevalence, time of onset and association with risk factors in 300 consecutive patients with acute chest pain and angiographically documented coronary artery disease. Eur Urol 2003; 44:360-5

22 Vlachopoulos C, Rokkas K, loakeimidis N, et al. Prevalence of asymptomatic coronary artery disease in men with vasculogenic erectile dysfunction: a prospective angiographic study. Eur Urol 2005;48:996-1003.

23 Mulhall J, Teloken P, Barnas J et al. Vasculogenic erectile dysfunction is a predictor of abnormal stress echocardiography. J Sex Med 2009;6:820-5

24 Hodges LD, Kirby M, Solanki J, et al. The temporal relationship between erectile dysfunction and cardiovascular disease. Int J Clin Pract 2007;61:2019-25.

25 Inman BA, Sauver JL, Jacobson DJ, et al. A population-based, longitudinal study of erectile dysfunction and future coronary artery disease. Mayo Clin Proc 2009:84:108-13.

26 Ponholzer A, Temml C, Obermayr R, et al. Is erectile dysfunction an indicator fo increased risk of coronary heart disease and stroke? Eur Urol 2005:48:512-8.

27 Thompson IM, Tangen CM, Goodman PJ, et al. Erectile dysfunction and subsequent cardiovascular disease. JAMA 2005:294:2996-3002.

28 Banks E, Joshy G, Abhayaratna WP, et al. Erectile dysfunction severity as a risk marker for cardiovascular disease hospitalisation and all-cause mortality: a prospective cohort study. PLoS Med 2013;10:e1001372.

29 Lewis RW, Fugl-Meyer KS, Corona G, et al. Definitions/epidemiology/risk factors for sexual dysfunction. J Sex Med 2010;7:1598-607.

30 Yaman 0, Gulpinar 0, Hasan T, et al. Erectile dysfunction may predict coronary artery disease: relationship between coronary artery calcium scoring and erectile dysfunction severity. Int Urol Nephrol 2008;40:117-23. 
31 Montorsi P, Ravagnani PM, Galli S, et al. Association between erectile dysfunction and coronary artery disease. role of coronary clinical presentation and extent of coronary vessels involvement: the COBRA trial. Eur Heart J 2006;27:2632-9.

32 Montorsi P, Ravagnani PM, Galli S, et al. Association between erectile dysfunction and coronary artery disease:matching the right target with the right test in the right patient. Eur Urol 2006;50:721-31.

33 Yassin AA, Saad F. Testosterone and erectile dysfunction. J Androl 2008;29:593-604.

34 Khera M. Androgens and erectile function: a case for early androgen use in postprostatectomy hypogonadal men. J Sex Med 2009;6:234-8.

35 Aversa A, Isidori AM, De Martino MU, et al. Androgens and penile erection: evidence for a direct relationship between free testosterone and cavernous vasodilation in men with erectile dysfunction. Clin Endocrinol 2000;53:517-22.

36 Wespes E, Amar E, Hatzichristou D, et al. EAU guidelines on erectile dysfunction: an update. Eur Urol 2006;49:806-15.

37 U.S. Food and Drug Administration. FDA Drug Safety Communication: fda cautions about using testosterone products for low testosterone due to aging; requires labeling change to inform of possible increased risk of heart attack and stroke with use. www. fda.gov/Drugs/DrugSafety/ucm436259.htm.

38 Bassil N, Alkaade S, Morley JE. The benefits and risks of testosterone replacement therapy: a review. Ther Clin Risk Manag 2009;5:427-48.

39 Søe KL, Søe M, Gluud C. Liver pathology associated with the use of anabolicandrogenic steroids. Liver 1992;12:73-9.

40 Randrup E, Baum N, Feibus A. Erectile dysfunction and cardiovascular disease. Postgrad Med 2015;127:166-72.

41 Wrishko R, Sorsaburu S, Wong D, et al. Safety, efficacy, and pharmacokinetic overview of low-dose daily administration of tadalafil. J Sex Med 2009:6:2039-48.
42 Seftel AD, Sun P, Swindle R. The prevalence of hypertension, Hyperlipidemia, diabetes mellitus and depression in men with erectile dysfunction. J Urol 2004; 171:2341-5

43 American association of sexuality educators, counselors and therapists https://www. aasect.org/certification

44 Kaplan HS. Basic principles of sex therapy, Plenum Publishing, 1980.

45 Randrup E, Wilson S, Mobley D, et al. Clinical experience with Mentor Alpha inflatable penile prosthesis. Report on 333 cases. Urology 1993;42:305-8.

46 Khera M, Albersen M, Mulhall JP. Mesenchymal stem cell therapy for the treatment of erectile dysfunction. J Sex Med 2015;12:1105-6.

47 Frey A, Sønksen J, Fode M. Low-intensity extracorporeal shockwave therapy in the treatment of postprostatectomy erectile dysfunction: a pilot study. Scand J Urol 2016;50:1-5.

48 Soni SD, Song W, West JL, et al. Nitric oxide-releasing polymeric microspheres improve diabetes-related erectile dysfunction. J Sex Med 2013;10:1915-25.

\section{Answers}

1. True

2. True

3. False (both groups have endothelial dysfunction)

4. True

5. True 